\title{
La comprensión lectora de textos técnicos en inglés y uso de técnicas pedagógicas interactivas para el aprendizaje en los estudiantes universitarios.
}

\section{Reading comprehension of technical texts in English and use of interactive pedagogical techniques for learning in university students.}

Rocío de los Ángeles Barragán Murilloํㅡㄹ Zoila Victoria Herrera Andrade ${ }^{2}$, Esthela Isabel Colcha Guashpa ${ }^{3}$, Daniela Fernanda Guano Merino ${ }^{4}$

\begin{abstract}
DOI: https://doi.org/10.33262/cienciadigital.v3i3.2.712

The purpose of this research is to analyze interactive pedagogical techniques in improving reading comprehension of technical texts in English for third level students of the School of Industrial Engineering of Escuela Superior Politécnica de Chimborazo in the city of Riobamba, Province of Chimborazo, in order to demonstrate the importance of learning English in future professionals through the use of these techniques to be recruited at work positions, considering that English language proficiency is essential for recruitment. The research designed is non-experimental, applied, correlational, field and bibliographic methodology is used. The methods used for the research process were scientific, analytical-synthetic and statistical. To collect the information, the survey and interview were used as instruments. The study population is made up of authorities, teachers and students; applying the survey to 104 students who mostly belong to the Costa and Amazonia Region of the third level that constitute the total population and the interview with 5 English teachers of the mentioned school and 4 authorities of the institution. For the processing of the data, descriptive and inferential statistics were used, where the tabulation of the information evidenced the students' reading deficiency in English. It was also determined that teachers know little about interactive techniques and the need for a guide that contains these types of techniques. For the validation and confidence of the instruments applied, the judgment of the experts in the area of English was used. To support the research process, a guide with interactive techniques was designed. We conclude that the use of
\end{abstract}

\footnotetext{
${ }^{1}$ Escuela Superior Politécnica de Chimborazo: Docente Inglés ESPOCH. Chimborazo Ecuador, obarragan@espoch.edu.ec

${ }^{2}$ Escuela Superior Politécnica de Chimborazo: Docente del Centro de Idiomas ESPOCH. Chimborazo Ecuador, zherreraa@ hotmail.com

${ }^{3}$ Escuela Superior Politécnica de Chimborazo: Docente de Inglés ESPOCH. Chimborazo Ecuador, e_colcha@espoch.edu.ec

${ }^{4}$ Escuela Superior Politécnica de Chimborazo: Docente Ingles ESPOCH. Chimborazo Ecuador, danielaguano@hotmail.es
} 
this didactic guide will contribute positively to the students' learning of the English Language.

Keywords: Reading Comprehension - Reading Technical Texts - Interactive Techniques - Interactivity - English.

\section{Resumen}

La presente investigación tiene como objetivo analizar las técnicas pedagógicas interactivas en el mejoramiento de la comprensión lectora de textos técnicos en inglés para los estudiantes de tercer nivel de la Escuela de Ingeniería Industrial de la Escuela Superior Politécnica de Chimborazo de la ciudad de Riobamba, Provincia de Chimborazo, con el fin de evidenciar la importancia que tiene el aprendizaje del idioma inglés en los futuros profesionales mediante el uso de estas técnicas para ser reclutados en los puestos laborales al considerar requisito indispensable el dominio del idioma inglés para su reclutamiento. La investigación diseñada es no experimental, se emplea metodología de tipo aplicada, correlacional, de campo y bibliográfica. Los métodos utilizados para el proceso investigativo fueron el científico, analítico-sintético y estadístico. Para la recolección de la información se empleó la encuesta y la entrevista como instrumentos. La población de estudio lo conforman autoridades, docentes y estudiantes; aplicando la encuesta a 104 estudiantes los cuales en su mayoría pertenecen a la Región Costa y Amazonia del tercer nivel que constituyen la población total y la entrevista a 5 docentes de inglés de la mencionada escuela y a 4 autoridades de la institución. Para el procesamiento de los datos se utilizó la estadística descriptiva e inferencial, en donde al tabular la información se evidenció la deficiencia de lectura en inglés de los estudiantes; también se determinó que los maestros conocen poco sobre técnicas interactivas y la necesidad de contar con una guía que contenga este tipo de técnicas. Para la validación y confianza de los instrumentos aplicados se usó el juicio de las expertas en el área de inglés. Como apoyo al proceso investigativo se diseñó una guía con técnicas interactivas, concluyendo que el uso de esta guía didáctica contribuirá positivamente en el aprendizaje del Idioma Inglés de los estudiantes.

Palabras Claves: Comprensión Lectora - Lectura Textos Técnicos - Técnicas Interactivas - Interactividad - Inglés.

\section{Introducción}

En la actualidad, la educación superior juega un papel importante en la generación de nuevos conocimientos a fin de satisfacer los requerimientos de la sociedad. Todo lo que se ve, se lee o se escucha está en inglés, convirtiéndose en parte del desarrollo formativo de cualquier persona.

Comprender el idioma inglés abre muchas posibilidades tanto a nivel escolar como profesional y la necesidad de dominarlo es cada vez más imperante. Una de las destrezas del idioma inglés 
es la lectura tema de estudio de esta investigación, pues los estudiantes deben dominar el idioma inglés porque su perfil profesional así se lo exige y una de las destrezas que requieren para poder manejar manuales, libros, etc., especializados es la comprensión lectora.

En base al estudio propuesto en esta investigación se observa que el nivel lector de estos estudiantes es deficiente, no son capaces de hacer inferencias o analizar textos; así también los docentes de inglés se encuentran desactualizados en sus conocimientos pedagógicos. No obstante, sienten interés y preocupación por el aprendizaje de sus estudiantes.

El uso de técnicas interactivas para desarrollar la comprensión lectora permite el trabajo colaborativo puesto que el estudiante debe ser un participante proactivo, mantiene el interés y la motivación, interactuando de forma permanente en la construcción conjunta del conocimiento.

Los cambios que se han producido con el desarrollo tecnológico, las TIC y dominio del idioma inglés, contribuyen a que el estudiante cambie la forma de aprender a través de herramientas aplicadas en el internet y redes sociales en donde los jóvenes dedican gran parte de su tiempo a estas actividades.

El autor Hernández (2006) señala "La importancia de los estilos de aprendizaje en la enseñanza del inglés como lengua extranjera mediante el uso del internet ha generado cambios significativos en esta nueva forma de interactuar y aprender", es decir que con la aparición del internet en la década de los 90, el mundo cambió para siempre y con ello la forma como se recibe la información, la educación, los negocios, etc.

El rol del docente es fundamental para el aprendizaje, pues debe iniciar con la selección de un modelo pedagógico, en base al tipo de aprendizaje que desea crear en sus estudiantes y lograr en ellos interés para que sea más fácil la tarea de la enseñanza. (Pérez Rodríguez, 2004) expone que la motivación como elemento muy importante en el uso de tecnologías en clase, contribuye a que los estudiantes aparte de sentirse motivados y comprometidos, se apropian de su propio aprendizaje sobre todo porque están activos fuera del aula de clases.

En este trabajo se presenta la Web 2.0 y sus herramientas web y recursos multimedia como técnicas pedagógicas interactivas para el aprendizaje en los estudiantes universitarios con el fin de desarrollar competencias pedagógico-digitales que permitan lograr mayor calidad en la comprensión lectora.

\section{Comprensión Lectora}

Para el autor Solís (2015), la comprensión lectora se define como el proceso por medio del cual un lector construye, a partir de su conocimiento previo, nuevos significados al interactuar con el texto. Esto es el fundamento de la comprensión: la interacción del lector con el texto. Esta es la base del entendimiento: la interacción del lector con el texto. Este proceso se desarrolla de manera diferente en cada lector, en razón de que cada individuo desarrolla diferentes esquemas y usa diferentes destrezas y habilidades cuando enfrenta un texto. 


\section{Habilidades de comprensión}

Las habilidades lingüísticas son entendidas como las aptitudes que permiten comunicarnos a través del saber: escuchar, hablar, leer y escribir. El escuchar es necesario y sirve para oír, comprender y entender las ideas de lo que nos dicen y lo que los otros quieren decirnos; el hablar, sirve para emitir, expresar nuestras ideas, sentimientos e inquietudes a través de la palabra; en tanto que la habilidad de la lectura sirve para comprender; pues el lector debe decodificar los mensajes, interpretarlas y comprenderlos; mientras que la habilidad escribir, permite expresar a través de ideas o símbolos, de acuerdo a la cultura para ser comprendidos por otros. (Araníbar Escarcha, N. R. , 2017)

\section{Condicionantes para la comprensión lectora}

Resulta casi imposible separar el concepto de lectura con el de comprensión lectora, ya que ambos se basan en los mismos procesos diferenciándose sólo en la presencia de un lector ideal, el cual determina una comprensión eficaz. Solís (2015), manifiesta que mientras más sea el conocimiento previo del lector, más probabilidades tiene de entender las palabras relevantes, realizar las inferencias correctas durante la lectura y elaborar correctamente los modelos de significado.

En este contexto, se incorporan las tecnologías de información y comunicación a través del uso de páginas de internet, correo electrónico y Weblog, apoyados en la metodología CALL (computer-assisted language learning), específicamente $\mathrm{CMC}$, la cual señala que el uso de la comunicación mediada por computadores puede favorecer el desarrollo de las habilidades comunicativas (Quintanilla, E. A., \& Ferreira Cabrera, A., 2010); su objetivo es mejorar el aprendizaje en aquellos que aprenden un idioma a través de medios computacionales, permitiendo que el aprendiz desarrolle, expanda y refine sus habilidades comunicacionales en una nueva lengua.

\section{Teorías del Aprendizaje}

El Constructivismo se fundamenta en la filosofía, psicología, sociología y educación. Esta teoría se basa en que el aprendizaje humano se construye, que la mente de los seres humanos crea nuevos conocimientos partiendo de conocimientos previos. El estudiante es un ser activo que a través de su participación activa le permite su aprendizaje; tiene control y le permite construir el conocimiento a través de las experiencias y estas crean esquemas. Estos esquemas son modelos mentales que guardamos en nuestras mentes los cuales van cambiando, agrandando o mejorando a través de los siguientes procesos: Asimilación y Alojamiento. (Piaget, 1969)

En cuanto a la tecnología con el aprendizaje constructivista Hernández (2008) menciona que los ordenadores crean un ambiente adecuado para que los alumnos expresen y muestren lo que 
han aprendido. Los docentes constructivistas promueven el uso de las TIC con sus estudiantes a diferencia de los tradicionales que no permiten que la creatividad de los estudiantes fluya evitando que piensen con libertad.

El Cognotivismo según los autores Pacciola y otros (2012) esta corriente considera que la mente no se limita a recibir información proveniente de los estímulos de fuera sino más bien la considera como un colaborador activo que constantemente verifica la congruencia entre el propio proyecto comportamental y las condiciones objetivas existentes distinguiendo de ellas las informaciones y autocorrigiéndolas. Considera al hombre en sus distintas dimensiones psicológica, evolutiva, clínica, biológica y sociológica.

Vigotsky considera el aprendizaje una pieza clave del desarrollo. En su opinión, la mejor enseñanza es la que se adelanta al desarrollo. En el modelo de aprendizaje que aporta siendo el contexto extremadamente importante para el aprendizaje. La teoría de Vigotsky se refiere a cómo el ser humano ya trae consigo un código genético o "línea natural del desarrollo" también llamado código cerrado, la cual está en función de aprendizaje, en el momento que el individuo interactúa con el medio ambiente. Su teoría toma en cuenta la interacción sociocultural. (Bermúdez, 2008)

El Aprendizaje significativo, según Méndez (2008) haciendo referencia a Ausbel dice que:

"Es un proceso por medio del que se relaciona nueva información con algún aspecto ya existente en la estructura cognitiva de un individuo y que sea relevante para el material que se intenta aprender. El aprendizaje debe necesariamente tener significado para el estudiante, si queremos que represente algo más que palabras o frases que repite de memoria en un examen". (p. 95)

Es una teoría que trata de los procesos que una persona usa para aprender particularmente en el aula, la naturaleza del aprendizaje, las condiciones, resultados y evaluación del aprendizaje. El aprendizaje significativo trata con los elementos, factores, condiciones de la adquisición y retención del conocimiento que se le ofrece al estudiante para que su aprendizaje sea significativo. (García C., 2012)

\section{El Rol Docente}

(Arias Vera, 2009) manifiesta que todo docente dentro de su actividad profesional está en la obligación de conocer los modelos pedagógicos que orientan su desempeño. Cada institución educativa tiene un modelo educativo y bajo este sus docentes deberán llevar a cabo el proceso de enseñanza aprendizaje.

El rol del docente dependerá de la situación. En algunas ocasiones deberá ser un controlador, un organizador, un tutor, un observador, un recurso, un participante, un apuntador o asesor; dependerá de la situación y las necesidades del estudiante el rol del docente. El docente debe 
desarrollar su actividad teniendo siempre en cuenta la pedagogía, ya que esta estudia a la educación como fenómeno complejo y multireferencial, lo que indica que existen conocimientos provenientes de otras ciencias y disciplinas que le pueden ayudar a comprender lo que es la educación; ejemplos de ello son la historia, la sociología, la psicología y la política, entre otras (Ávila, 2010).

\section{El Docente en el Siglo XXI}

Con la globalización en las últimas décadas es necesario tomar en cuenta al individuo como tal. Aquí nace la era o sociedad del conocimiento. Una era en la que no es necesario guardar la información en el cerebro, se hace uso de celulares, computadores, tablets, libros, etc. Se requiere que el estudiante analice, procese y argumente la información no que se limite a memorizar. Pese a los constantes cambios del mundo actual, la escuela sigue con currículos antiguos. Para superar esto: el docente deberá contribuir a formar individuos más inteligentes cognoscitivamente, sociales, afectivos, estéticos y práxicos. (De Zubiría Z., 2013)

(Domínguez C., 2012) expresa: El docente con respecto a las nuevas tecnologías, para que el proceso educativo se dé exitosamente, deberá ser: diseñador gráfico, asesor, comunicador, orador y evaluador. Adicionalmente, debe escoger las herramientas adecuadas para que el alumno haga uso de ellas y resuelva problemas que contribuyan a su formación integral.

Tanto docentes como estudiantes del siglo XXI deben contar con destrezas que ni siquiera se lo hubieran imaginado, los estudiantes de este siglo se encuentran justo ahora sentados en un aula de clase listos para explorar, diseñar y crear. Es responsabilidad del docente proporcionarles de los recursos necesarios integrando la tecnología.

\section{La Web 2.0}

El señor Tim O'Reilly nació en Irlanda, teniendo su desarrollo y crecimiento en San Francisco de los Estados Unidos, fue el fundador de la editorial de libros de informática O'Reilly Media, es conocido por haber acuñado el término Web 2.0, término que apareció en el año 2004.

La Web 2.0 surge en contraste a la web 2.0 ofreciendo posibilidades diferentes a Altavista, correo de Hotmail, encarta o Netscape navigator 4.7, el contenido de la web 2.0 es el resultado de un trabajo colectivo contribuyendo al pensamiento crítico, autonomía, iniciativa, trabajo colaborativo y responsabilidad individual. (Gónzalez, 2015)

La Web 2.0 es el resultado de varias evoluciones ocurridas al mismo tiempo:

- El Internet avanzó al punto de que la conectividad es posible en cualquier parte del mundo.

- Las laptops se convirtieron en un artefacto que se encuentra en la mayoría de los hogares, escuelas y oficinas. 
- Los teléfonos móviles se convirtieron en dispositivos a través de los cuales se pueden hacer llamadas y acceder al Internet.

- Los navegadores se volvieron ampliamente disponibles y fáciles de usar.

- Las aplicaciones web se desarrollaron para que puedan ser usadas en diferentes dispositivos.

- Los desarrolladores web usaron la web para usar nuevas versiones de aplicaciones software.

- La sociedad se adaptó a estos cambios tecnológicos.

Las formas de conectarse al Internet han ido evolucionando, desde el uso de los teléfonos del hogar en el pasado cuyo servicio no era costoso hasta lo que actualmente se usa fibras ópticas, satélites, cable, etc. Los medios que apoyan para el uso de la Web 2.0 son: Tecnología, Redes sociales, Sindicación de contenidos, Servicios web y Software del servidor (Fernández Martín, s.f.)

Las herramientas Web 2.0 son un recurso innovador y efectivo en la lectura. Vocabulario, fluidez y comprensión de textos se pueden mejorar a través del uso de ambientes colaborativos. (Banister, 2008)

\section{Lectura de Textos Técnicos}

Arrieta de Meza y otros (2008) señalan que:

"La lectura es un proceso perceptivo, lingüístico y cognitivo que comienza en un nivel perceptivo y termina en un nivel conceptual. Una buena decodificación lectora es previa a la compresión, la cual puede concebirse como el entendimiento del significado de un texto y la intencionalidad del autor”. (p. 39)

Fajardo Hoyos y otros (2012) manifiestan que la lectura es una herramienta para tener acceso a la información, la cultura y el aprendizaje. Se considera una habilidad compleja en la que intervienen procesos de codificación, recodificación y compresión:

- El proceso de codificación: es el análisis visual del texto.

- Los procesos de recodificación: son los procesos fonológicos que son una forma de representación temporal de la información basada en fonemas. La codificación y recodificación permiten el acceso léxico; en otras palabras, reconocimiento de palabras.

- Los procesos de comprensión: es el análisis sintáctico y semántico del texto.

La lectura es una destreza multifacética y compleja y siempre ha sido de constante revisión el cómo enseñar a leer, especialmente con el auge del Internet. Diferentes estrategias de lectura se requieren cuando se trata de textos electrónicos. La forma del texto en si ha cambiado, con los nuevos géneros de texto que han aparecido, por ejemplo, email, chat, blogs, etc., así como nuevas combinaciones de texto e imagen. Todos estos nuevos textos requieren nuevas destrezas. 


\section{Técnicas Interactivas}

La interactividad está ligada al aprendizaje efectivo. Interactuar con otras personas o con información ayuda a entender conceptos, resolver problemas y retener información. Se podría pensar que interacción hace referencia a hacer preguntas y contestarlas, pero existe una variación de modelos de interacción.

Las nuevas generaciones de estudiantes universitarios han crecido en un ambiente altamente interactivo, por lo que se espera que el aprendizaje sea interactivo. El aprendizaje en un proceso interactivo en el cual el estudiante desarrolla su comprensión integrando hechos, experiencia y prácticas.

Aparici y Silva (2012) manifiestan que los principios de interactividad son tres:

- Participación-intervención. - no se limita a dar una respuesta positiva o negativa, el usuario interfiere, interviene en el contenido de la información o modifica el mensaje.

- Bidireccional-hibridación. - se refiere a la que la comunicación es la producción de la emisión y la recepción, codificar y decodificar se convierten en un solo elemento

- Permutabilidad-potencialidad. - la comunicación supone múltiples redes que se encuentran articuladas con conexiones y la libertad para poder hacer cambios, asociaciones y producir diversos significados

Este autor manifiesta que en base a estos fundamentos el entendimiento de la transmisión pierde su lógica puesto que abre espacios para la participación sensomotora y semántica. Los usuarios no se conforman con tan solo recibir, leer u oír información, sino que desean interactuar, convertirse en sujetos de información. El uso de la interactividad en la enseñanza, requiere compromiso por parte de las universidades, es decir, apoyo a los docentes, infraestructura y evaluación permanente.

\section{Idioma Inglés}

Quezada (2005), afirma que:

El auge del inglés en este siglo ha sido considerable. Esto se debe a las tendencias globales en tecnología, demografía y economía. Dado que el inglés es uno de los idiomas más usados alrededor del mundo, muchas veces se hace referencia a él como el "idioma global", la "lengua franca" de la era moderna. Cientos de millones de personas están aprendiendo inglés, el idioma del planeta para el comercio, la tecnología y, cada vez más, para el poder.

El idioma ingles ha adquirido el status de lengua franca debido al colonialismo Británico y el americanismo particularmente de los avances y dominio de la post guerra Estados Unidos en 
economía, tecnología y ciencia, educción e incluso entretenimiento. Las personas se comunican con sus socios de negocios en inglés, escuchan música pop anglófona, miran novelas o películas americanas, leen artículos, navegan en internet el cual es casi $80 \%$ en inglés. (Luo, W. , 2007 )

\section{Importancia del idioma Inglés}

Noam Chomsky (1965), citado en Sarmiento, 1996 afirma que los seres humanos tenemos la capacidad innata para adquirir el lenguaje que no se aplica únicamente al aprendizaje de la lengua materna sino a todas las lenguas y que la función que cumple el ambiente que rodea al niño no es la de enseñar directamente, sino la de "activar la capacidad innata y convertirla en competencia lingüística" (Sarmiento, 1996, p. 292). Se justifica esta idea al saber que nunca es demasiado pronto para exponer a un niño a la segunda lengua en razón de que desde que nace presenta la capacidad para aprenderla.

El idioma inglés posee un léxico muy rico en el cual se superponen palabras de origen germánico y sinónimos latinos, complicada fonética, ortografía y preposiciones.

\section{Metodología}

Para el diseño de investigación del presente estudio se procedió a la revisión bibliográfica del autor Toledo (2012), tomando como guía su libro Metodología de la Investigación, para llevar a la práctica este estudio.

El diseño de la investigación es No Experimental la cual nos permitió observar y conseguir la información para su posterior análisis.

El tipo de investigación se fundamenta por el propósito: aplicada, por el nivel: correlacional, por el lugar: de campo y por el origen: bibliográfica. Los métodos planteados son científico, analìtico-sintètico y estadístico

La población objeto de estudio está compuesta por 104 estudiantes del tercer nivel de la Carrera de Ingeniería Industrial de la Escuela Superior Politécnica de Chimborazo, 5 docentes de inglés que imparten clases en esta escuela y 4 autoridades de la ESPOCH registrando un total de 113.

Las técnicas utilizadas para obtener la información son la encuesta y la entrevista, para lo cual se estructura un cuestionario para aplicarlo a los estudiantes y una guía de preguntas abiertas dirigida a las autoridades y docentes de la institución.

La información obtenida es clasificada, tabulada y analizada, datos que se formaliza en cuadros y representaciones gráficas de cada una de las preguntas en el programa Microsoft Excel. 


\section{Resultados}

Los resultados que se presentan son obtenidos de la aplicación de las entrevistas con preguntas abiertas a 5 docentes de inglés y a 4 autoridades de la institución y de las encuestas a 104 estudiantes de tercer nivel de inglés de la Escuela de Ingeniería Industrial de la Escuela Superior Politécnica de Chimborazo. En donde se presentan del total de preguntas formuladas en la encuesta las preguntas $4,5,8,9,11$ y 22 consideradas las más representativas, para la entrevista a las autoridades y docentes se considera todas las preguntas para su respectivo análisis.

4.- ¿Según su criterio, cree usted que los docentes de inglés conocen sobre Pedagogía y Andragogía?

\section{Cuadro 1 Pregunta 4}

\begin{tabular}{lll}
\hline \multicolumn{1}{c}{ RESPUESTAS } & FRECUENCIA & PORCENTAJE \\
\hline Mucho & 5 & 5 \\
Medianamente & 54 & 52 \\
Poco & 38 & 36 \\
Nada & 7 & 7 \\
\hline Total & 104 & 100 \\
\hline
\end{tabular}

Elaborado por: Secretaría de la Facultada de Idiomas ESPOCH

Análisis: Del 100\% de la población encuestada, el 52\% de ellos considera que los docentes de inglés conocen medianamente sobre Pedagogía y Andragogía, el 36\% afirma que los docentes conocen poco, el 7\% de los docentes conocen nada y el 5\% consideran que los docentes están capacitados. Esto significaría que a pesar de no ser los estudiantes profesionales en la educación, se encuentran en capacidad de conocer y entender términos sobre enseñanza como es el caso de Pedagogía y Andragogía. En lo que respecta a esta pregunta, está muy clara la falta de preparación sobre pedagogía por parte del profesorado.

5.- ¿Considera usted que la didáctica que emplean los docentes de inglés es adecuada?

\section{Cuadro 2 Pregunta 5}

\begin{tabular}{lll}
\hline RESPUESTAS & FRECUENCIA & PORCENTAJE \\
\hline Mucho & 2 & 2 \\
Medianamente & 50 & 48 \\
Poco & 43 & 41 \\
Nada & 9 & 9 \\
\hline Total & 104 & 100 \\
\hline
\end{tabular}

Elaborado por: Secretaría de la Facultada de Idiomas ESPOCH 
Análisis: Del 100\% de la población encuestada, el 48\% de los encuestados manifiestan que la didáctica empleada por los docentes de inglés es medianamente adecuada, el $41 \%$ afirma que es poco adecuada el $9 \%$ responde con nada adecuada y en el $2 \%$ considera que la didáctica es adecuada. Estos resultados revelan que para la gran mayoría de los estudiantes la forma de enseñar de los docentes no es la más apropiada coincidiendo con el punto analizado anteriormente se debería utilizar documentos que faciliten mejorar la didáctica en el aula. 8.- ¿A su criterio el docente de inglés facilita el aprendizaje del idioma?

\section{Cuadro 3 Pregunta 8}

\begin{tabular}{lll}
\hline RESPUESTAS & FRECUENCIA & PORCENTAJE \\
\hline Mucho & 11 & 11 \\
Medianamente & 50 & 48 \\
Poco & 38 & 36 \\
Nada & 5 & 5 \\
\hline Total & 104 & 100 \\
\hline
\end{tabular}

Elaborado por: Secretaría de la Facultada de Idiomas ESPOCH

Análisis: Del 100\% de la población encuestada, el 48\% de los encuestados consideran que los docentes facilitan medianamente el aprendizaje, el 36\% considera que el docente facilita el proceso de aprendizaje poco, el $11 \%$ consideran que si cumple como facilitador el docente y el $5 \%$ responde con nada. Se puede concluir según la opinión de la mayoría de los estudiantes, que el docente no logra cumplir como facilitador del aprendizaje, razón por la cual se podría interpretar que el docente necesita actualización sobre su materia.

9.- ¿Cuándo se le presenta un texto escrito, cree usted que las actividades propuestas facilitan la comprensión lectora?

\section{Cuadro 4 Pregunta 9}

\begin{tabular}{lll}
\hline RESPUESTAS & FRECUENCIA & PORCENTAJE \\
\hline Mucho & 18 & 17 \\
Medianamente & 36 & 35 \\
Poco & 44 & 42 \\
Nada & 6 & 6 \\
\hline Total & 104 & 100 \\
\hline
\end{tabular}

Elaborado por: Secretaría de la Facultada de Idiomas ESPOCH 
Análisis: Del 100\% de la población encuestada, el 42\% responden que las actividades que van junto con el documento escrito contribuyen medianamente a desarrollar la comprensión lectora, el $35 \%$ contribuyen poco, el $17 \%$ afirma que si contribuyen las actividades y el $6 \%$ nada. Concluyendo que los estudiantes ratifican la falta de actividades que faciliten la comprensión lectora, para lo cual sería importante desarrollar actividades que se incluyan en la enseñanza del idioma inglés para mejora la comprensión de la lectura de los textos en este idioma.

11.- ¿Conoce usted técnicas interactivas para el aprendizaje del idioma inglés?

\begin{tabular}{lll} 
Cuadro 5 Pregunta 11 & & \\
\hline RESPUESTAS & FRECUENCIA & PORCENTAJE \\
\hline Mucho & 4 & 4 \\
Medianamente & 40 & 39 \\
Poco & 40 & 38 \\
Nada & 20 & 19 \\
\hline Total & 104 & 100 \\
\hline
\end{tabular}

Elaborado por: Secretaría de la Facultada de Idiomas ESPOCH

Análisis: Del 100\% de la población encuestada, el 39\% de los encuestados conoce medianamente sobre técnicas interactivas para el aprendizaje del idioma inglés, el 38\% afirma que conoce poco sobre técnicas interactivas, el $19 \%$ responde con nada sobre el tema y el $4 \%$ De la información obtenida sobre el tipo de técnicas, se observa que los estudiantes conocen medianamente poco y nada lo que significa que los docentes deben reforzar en clases sobre el tipo de técnicas interactivas para una mejor comprensión e interacción del aprendizaje basad en la tecnología.

22.- ¿Con que frecuencia el docente de inglés utiliza los entornos virtuales para desarrollar la comprensión lectora?

\section{Cuadro 1 Pregunta 22}

\begin{tabular}{lll}
\hline RESPUESTAS & FRECUENCIA & PORCENTAJE \\
\hline Mucho & 1 & 1 \\
Medianamente & 25 & 24 \\
Poco & 52 & 50 \\
Nada & 26 & 25 \\
\hline Total & 104 & 100
\end{tabular}

Elaborado por: Secretaría de la Facultada de Idiomas ESPOCH

Análisis: Del 100\% de la población encuestada, se puede indicar que el 50\% de los estudiantes manifiestan que los docentes utilizan poco los entornos virtuales para desarrollar la lectura en inglés, el 25\% indican que los docentes no usan para nada estos entornos en la lectura, el $24 \%$ 
dice que se usa medianamente y el $1 \%$ responden que si usan los docentes. Con estas respuestas, se puede observar que los docentes están renuentes al uso de las nuevas tecnologías para desarrollar destrezas como la lectura en inglés, situacion que confirma el uso de recursos tradicionales como texto impreso, pizarrón y marcador.

En la entrevista a las autoridades se obtuvo las siguientes respuestas:

1. La comprensión lectora del idioma ingles es de gran importancia en la formación profesional de los estudiantes de ingeniería industrial, coincidiendo las autoridades en que los docentes de esta área requieren implementar mejores metodologías para desarrollar la comprensión lectora en sus estudiantes.

2. Responden que el nivel de inglés de los estudiantes no es óptimo, así mismo consideran que los docentes de inglés requieren implementar mejores metodologías para desarrollar la comprensión lectora.

3. La importancia que tiene la utilización de métodos interactivos para el aprendizaje.

4. Expresan que la institución no tiene jornadas de capacitación para los docentes en forma general peor aún sobre técnicas en comprensión lectora.

5. Así también se puede decir que la metodología que emplean los docentes debe ser actualizada y acorde a las nuevas exigencias de la educación.

6. En los estudiantes es necesario concienciar la importancia de la lectura para sus futuras carreras.

Análisis: En base a las respuestas obtenidas en la entrevista aplicada a las autoridades de la institución, están conscientes de las falencias existentes en sus docentes por lo que deberán apoyar a la creación e implementación de nuevos programas de capacitación que permita a sus profesionales estar más actualizados para un mejor desempeño en las áreas de la enseñanza en los estudiantes para beneficio de la sociedad.

En la entrevista a los docentes estas fueron sus respuestas:

1. Pese a que algunos de los maestros han mencionado el uso de recursos tecnológicos ninguno de ellos indica exactamente técnicas interactivas para la enseñanza del idioma.

2. Existe una idea equivocada o el desconocimiento de lo que es una técnica interactiva.

3. La mayoría de docentes indican que el nivel lector de sus estudiantes es muy bajo. Se requiere desarrollar mecanismo que propendan al desarrollo de esta destreza.

4. Mayoritariamente los docentes evidencian la falta de capacitación en inglés. Se pone de manifiesto la falta de gestión de las autoridades para capacitación sobre inglés.

5. La mayor parte de docentes opinan que una posible solución a la falta de comprensión lectora de los estudiantes podría ser una guía que incluya técnicas para desarrollar lectura dado que en la institución carece de bibliografía sobre el tema.

6. Todos concuerdan que es necesario tener acceso a internet, aulas virtuales, laboratorios de punta puesto que al momento el internet es deficiente y no existen laboratorios que abastezcan la población estudiantil. 
Análisis: Se puede concluir en base a las respuestas de la entrevista aplicada a los docentes, que estos profesionales no cuentan con un plan de capacitación propuesto por la institución para su actualización profesional, situacion que pone en desventaja la enseñanza aprendizaje, al no contar con recursos, técnicas y herramientas propias para el aprendizaje de sus estudiantes.

Los resultados obtenidos en base a las preguntas más relevantes planteadas en los instrumentos de recolección de información, evidencian que el nivel lector del grupo de estudiantes en esta investigación es deficiente, razón por la cual se propone contar con una guía con técnicas interactivas para desarrollar la comprensión lectora de textos técnicos en inglés para los estudiantes de tercer nivel de la Carrera de Ingeniería Industrial de la ESPOCH, el nivel de formación profesional debe estar actualizado y acorde a las nuevas tecnologías que permitirán a los estudiantes alcanzar a mejorar su destreza en la comprensión de textos en el idioma inglés.

\section{Validez y Confiabilidad}

Para la validación de los instrumentos aplicados se aplicó el juicio de tres expertos en el área y del manejo de instrumentos, quienes fueron: MSc. Adriana Cundar, MSc. Fanny Zambrano y MSc. Sandra Abarca, a quienes se les entregó la siguiente documentación:

- Oficio

- Instrucciones

- Preguntas directrices

- Objetivos

- Matriz de operacionalización de variables

- Instrumentos de Investigación (entrevistas para docentes y autoridades y encuestas para estudiantes)

- Formulario para la validación

Tanto para la validez como para la confiabilidad de los instrumentos de la investigación de campo, se usó el juicio de las expertas en el área de inglés antes mencionadas.

\section{Conclusiones}

- Los estudiantes no cuentan con técnicas de comprensión lectora que les permita generar hábitos de lectura y mejorar su nivel de inglés, situacion que se debe a que no han desarrollado correctamente las destrezas del idioma inglés durante su permanencia en la institución.

- Los docentes de inglés que laboran en esta escuela no utilizan técnicas interactivas para impartir sus clases, se limitan al uso de técnicas pedagógicas tradicionales. Estos docentes no cuentan al momento con capacitación de ningún tipo.

- No existe ningún tipo de capacitación por parte de las autoridades para los docentes sobre metodología. Las autoridades de Ingeniería Industrial se encuentran preocupadas 
por el bajo nivel del estudiantado en inglés, considerando que los mismos no han tomado medidas correctivas para solventar esta deficiencia.

\section{Referencias Bibliográficas}

Adam, F. (1970). Andragogía ciencia en la educación para adultos . Caracas: FIDEA.

Aparici, R., \& Silva, M. . (2012). www.revistacomunicar.com. Obtenido de http://www.revistacomunicar.com

Apostilla. (2014). "Concepto definiciones . Educacion P -definista.

Araníbar Escarcha, N. R. . (15 de 08 de 2017). uatf.edu.bo. Obtenido de uatf.edu.bo: http://www.uatf.edu.bo

Arias Vera, C. E. (2009). Fundamentos Pedagógicos De La Práctica Docente Desde La Academia De Platón Hasta La Ética En El Modelo Por Competencias. Obtenido de file:///C:/Users/Usuario/Downloads/Dialnet-

FundamentosPedagogicosDeLaPracticaDocenteDesdeLaAc-4953807.pdf

Arias, V. C. (2009). Fundamentos Pedagógicos De La Práctica Docente Desde La Academia De Platón Hasta La Ética En El Modelo Por Competencias. Retrieved from file:///C:/Users/Usuario/Downloads/Dialnet-.

Arrieta de Meza, B. (2008 ). Interferencia de los neologismos en la comprensión lectora de textos acádemicos. . http://www.redalyc.org/pdf/761/76111716003.pdf.

Ausubel, D. (1970). Teorias del Aprendizaje Significativo. Barcelona: Paidos.

Ávila, E. (2010). Desarrollo de la Inteligencia. . Riobamba: Publicaciones JG.

Banister, S. (2008). Web 2.0 Tools in reading classroom: . Teacher exploring literacy in the 21 st century. International Journay of Technology in Teaching and Learning, 109-116.

Batista, E. (2007). Lineamientos pedagógicos para la enseñanza y el aprendizaje. Colombia: Universidad de Colombia.

Bermúdez, R. (2008). Piscología Evolutiva del niño. . La Habana: CANECA.

Bonvecchio De Aruani, M. (2006). Evaluación de Los Aprendizajes. . Noveduc.

Cardona Ossa, G. (2002). Tendencias Educativas para el Siglo XXI Educación Virtual, Online y @learning Elementos para la discusión. Retrieved from http://www.edutec.es/revista/index.php/edutec-e/article/view/542/276.

Chomsky, N. (1965). La linguisttica. Massachuset: Santillana.

De Zubiría Z., J. (2013). Plandecenal.edu.co. (El maestro y los desafíos a la educación en el siglo XXI. ) Obtenido de http//.www.landecenal.edu.co 
Domínguez C., G. (2012). El Rol del Docente Ante la Aplicación de Tecnologías para los Procesos Formativos.

Doron, R., \& Parot, F. . (1998). Diccionario Akal de Psicología. AKAL.

Doron, R., \& Parot, F. . (1998). Diccionario Akal de Psicología. AKAL.

EF. (2014). ef. Obtenido de http://www.ef.com.ec

Fajardo Hoyos, A. (2012). Acceso léxico y comprensión lectora: Un estudio con jóvenes universitarios. Retrieved from http://redie.uabc.mx/redie/article/view/305/660.

Fernández Martín, E. (s.f.). (Web 2.0. Sindicación de contenidos (RSS).) Obtenido de https://ddd.uab.cat/pub/dim/16993748n8/16993748n8a1.pdf

García C., A. (2012). Las TIC en el aula: por un aprendizaje constructivo y significativo. Aplicación en alumnos de la USEE. García C., A. (2012). Las TIC en el aula: por un aprendizaje constructivo y significativo. Aplicación en alumnos de la USEE. Retrieved from

http://openaccess.uoc.edu/webapps/o2/bitstream/10609/24681/6/agarciacamPracticum 0613memoria.pdf.

Gónzalez, V. J. (2015). Nuevas tendencias en innovación educativa superior. . ACCI.

Graells, P. (2005). La Web 2.0 y sus aplicaciones didácticas. . Retrieved from Departamento de Pedagogía Aplicada, Facultad de Educadio UAB: : http://www.peremarques.net/web20.htm.

Hernández, R. L. (2006). Aprendiendo Inglés. . México.

Hernández, R. S. (2008). El modelo constructivista con las nuevas tecnologías: aplicado en el proceso de aprendizaje. Retrieved from http://www.uoc.edu/rusc/5/2/dt/esp/hernandez.pdf.

Icarito. (03 de 05 de 2012). http://www.icarito.cl.

Iglesias, M. (2006). Curso de Didáctica Aplicada. La Habana: Pueblo y Educación.

Luo, W. . (2007 ). researchbank.rmit.edu.au. Obtenido de https://researchbank.rmit.edu.au/eserv/rmit:9876/Luo.pdf

Luppicini, R. (2003). Towards a Cyber-Constructivist Perspective (CCP) of Educational Design. . Retrieved from http://www.cjlt.ca/index.php/cjlt/article/view/33/30.

Mata, F. S. (2007). Habilidades lingüísticas y comprensión lectora, una investigación empírica. . Retrieved from Dialnet: file:///C:/Users/Usuario/Downloads/DialnetHabilidadesLinguisticasYComprensionLectoraUnaInves-2533523.pdf.

Méndez, C. Z. (2011). uned. Obtenido de http://www.uned.ac.cr/ecsh

Méndez, Z. (2008). Aprendizaje y Cognición. . San José: Editorial UNED. 
Nafría, I. (2008). El usuario el nuevo rey de internet. España: Ediciones Gestión 2000.

Pacciola, A., \& Mancini, F. (2012). Cognitivismo existencial. Bogotá: San Pablo.

Piaget, J. (1969). Psicologia y Pedagogia: Teorias del aprendizaje . Barcelona: Planeta.

Quezada, C. . (2005). eumed.net. Obtenido de http://www.eumed.net

Quintanilla, E. A., \& Ferreira Cabrera, A. (2010). Habilidades Comunicativas en L2 mediatizadas por la Tecnología en el Contexto de los Enfoques por tareas y cooperativo. . Estudios Pedagógicos Valdivia, 213 - 231.

Ramírez Prado, M. E. (2009). La mediación en el proceso de enseñanza aprendizaje. Andalucia: La mediación en el proceso de enseñanza aprendizaje. Retrieved from http://www.csi-

csif.es/andalucia/modules/mod_ense/revista/pdf/Numero_14/ELENA_RAMIREZ_2.p df.

Ramírez, P. M. (2009). La mediación en el proceso de enseñanza aprendizaje. Ramírez Prado, M. E. (2009). La mediacihttp://www.csicsif.es/andalucia/modules/mod_ense/revista/pdf/Numero_14/ELENA_RAMIREZ_2.p df.

Rincón, P. M. (2011). El perfil docente ante la incorporación de las Tecnologías de la información y Comunicación (TIC) en la educación.

Sánchez, F. (13 de 05 de 2018). lifeder.com. Recuperado el 11 de 06 de 2019, de https:/www.lifeder.com/teorias-del-aprendizaje/

Solís, M. (2015). secure. Obtenido de secure: https://secure.urkund.com

Soriano, D. M. (2009). Los caminos de Paulo Freire en Córdoba. Córdoba: Eduvim.

Toledo Díaz, E. (2012). Elementos de la Metodología de la Investigación. La Habana: Pueblo y Educación.

UNESCO. (2008). Eduteka.org. Obtenido de http://www.eduteka.org/articulos/EstandaresDocentesUnesco 


\section{PARA CITAR EL ARTÍCULO INDEXADO.}

Barragán Murillo, R. de los Ángeles, Herrera Andrade, Z., Colcha Guashpa, E., \& Guano Merino, D. (2019). La comprensión lectora de textos técnicos en inglés y uso de técnicas pedagógicas interactivas para el aprendizaje en los estudiantes universitarios. Ciencia Digital, 3(3.2), 6-23. https://doi.org/10.33262/cienciadigital.v3i3.2.712

\section{LCiencia}

El artículo que se publica es de exclusiva responsabilidad de los autores y no necesariamente reflejan el pensamiento de la Revista Ciencia Digital.

El artículo queda en propiedad de la revista y, por tanto, su publicación parcial y/o total en otro medio tiene que ser autorizado por el director de la Revista Ciencia Digital.
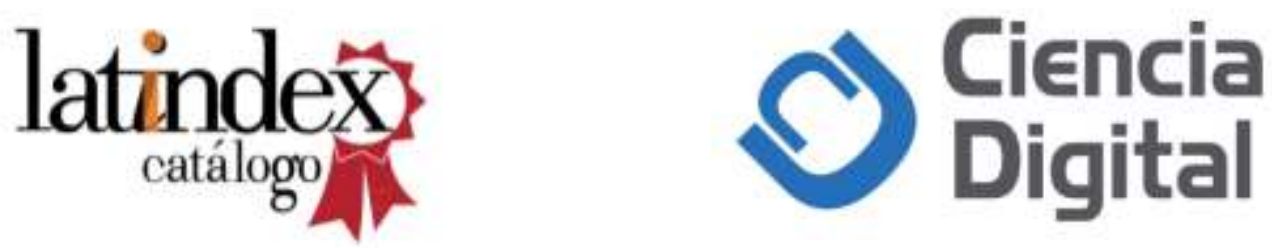